\title{
Observing wind, aerosol particles, cloud and precipitation: Finland's new ground-based remote-sensing network
}

\section{A. Hirsikko et al.}

Correspondence to: A. Hirsikko (anne.hirsikko@fmi.fi; a.hirsikko@fz-juelich.de) 
This supplement contains additional complementing material for the manuscript. Shown are technical details of Doppler and Raman lidars, and cloud radar (Tables S1-S3). Effect of telescope focus on Doppler lidar data acquisition is presented in Figure S1. Wind speed measured with Doppler lidar and sonic anemometer is compared in the Figure S2. 
Table S1. Technical details of the Doppler lidars by HALO Photonics (Pearson et al., J. Atmos. Ocean. Tech., 26, 240-250, 2009).

\begin{tabular}{|ll|}
\hline Input voltage & $230 \mathrm{~V} \mathrm{AC}$ \\
\hline Power supply output & $24 \mathrm{~V} \mathrm{DC}$ (includes UPS) \\
\hline Data and other connections & $2 \times \mathrm{RJ} 45,2 \mathrm{xUSB}$ \\
\hline Power consumption & $\begin{array}{l}\text { Nominal 140W (max 250W, when fully } \\
\text { cooling or heating) }\end{array}$ \\
\hline Pulses per ray & 15000, adjustable \\
\hline Pulse repetition frequency & $15 \mathrm{kHz}$ \\
\hline Fast Fourier Transform length & $\begin{array}{l}1024\left(3.8 \mathrm{~cm} \mathrm{~s}^{-1} \text { velocity resolution), }\right. \\
\text { adjustable }\end{array}$ \\
\hline Maximum Doppler velocity measurement & $\pm 19.2 \mathrm{~m} \mathrm{~s}^{-1}$ \\
\hline Velocity resolution & $0.038 \mathrm{~m} \mathrm{~s}{ }^{-1}$ \\
\hline Points per range gate (1 point $=3 \mathrm{~m})$ & $\begin{array}{l}10(\mathrm{vertical} \text { resolution } 30 \mathrm{~m}, \text { adjustable: } \\
\text { minimum } 18 \mathrm{~m})\end{array}$ \\
\hline Sample frequency & $50 \mathrm{MHz}$ \\
\hline Line of site maximum range & $9600 \mathrm{~m}$ \\
\hline Line of site minimum range & $90 \mathrm{~m}$ \\
\hline Focus setting & $\begin{array}{l}\text { Infinity, electronically adjustable (500m- } \\
\text { infinity) }\end{array}$ \\
\hline Heating set point & $15^{\circ} \mathrm{C}$ \\
\hline Cooling set point & $25^{\circ} \mathrm{C}$ \\
\hline Ambient operation specification & $-15^{\circ} \mathrm{C}$ to $+40^{\circ} \mathrm{C}$ \\
\hline
\end{tabular}


Table S2. Technical details of emitter and receiver of Polly ${ }^{\mathrm{XT}}$ Raman lidar.

\begin{tabular}{|c|c|c|}
\hline \multirow{6}{*}{ 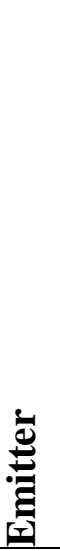 } & Laser type & Nd:YAG \\
\hline & Emitted energy per pulse $(\mathrm{mJ})$ & $180(1064 \mathrm{~nm}) ; 110(532 \mathrm{~nm}) ; 60(355 \mathrm{~nm})$ \\
\hline & Repetition frequency $(\mathrm{Hz})$ & 20 \\
\hline & Beam divergence (mrad) & $<0.2$ (after beam expansion) \\
\hline & Pulse duration (ns) & 6-8 (1064 nm); 6-8 (532 nm); 5-7 (355 nm) \\
\hline & Polarization orientation & Parallel $(532 \mathrm{~nm})$; vertical $(355 \mathrm{~nm})$ \\
\hline \multirow{9}{*}{ 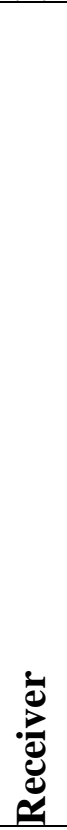 } & Telescope type & Newton, primary parabolic, flat folding mirror \\
\hline & Telescope aperture diameter (m) & 0.3 \\
\hline & Telescope obscuration diameter (mm) & 66 \\
\hline & Focal length (m) & 0.9 \\
\hline & Field of view (mrad) & 1 (changeable) \\
\hline & Fieldstop type & Circular aperture \\
\hline & Fieldstop size (mm) & 0.9 (changeable) \\
\hline & Telescope-laser axes distance $(\mathrm{m})$ & $\sim 0.2$ \\
\hline & Collimation focal length (mm) & 60 \\
\hline
\end{tabular}


Table S3. Technical details of Doppler cloud radar Mira 35S (Metek GmbH).

\begin{tabular}{|ll|}
\hline $\begin{array}{l}\text { Transmitter frequency } \\
\text { corresponding wavelength }\end{array}$ & $35.2 \mathrm{GHz}$ \\
\hline Transmitter pulse power & $8.5 \mathrm{~mm}$ \\
\hline $\begin{array}{l}\text { Pulse length } \tau \\
\text { corresponding range resolution }\end{array}$ & $30 \mathrm{~kW}$ \\
\hline $\begin{array}{l}\text { Pulse Repetition frequency (PRF) } \\
\text { corresponding velocity range }\end{array}$ & $100-400 \mathrm{~ns}$ (standard setting $\left.\tau_{0}=200 \mathrm{~ns}\right)$ \\
corresponding maximum range & $2.5-10 \mathrm{kHz}$ (standard $\left.\mathrm{PRF}_{0}=5 \mathrm{kHz}\right)$ \\
\hline Maximum duty cycle & $+/-5.3-+/-21.3 \mathrm{~m} / \mathrm{s}$ \\
\hline Maximum number of range gates & $58-13 \mathrm{~km}$ \\
\hline Minimum range: & $1: 500$ \\
\hline Antenna diameter & $2 * 1000($ co- and cross-channel) \\
corresponding beam divergence: & $150 \mathrm{~m}$, full sensitivity above $300 \mathrm{~m}$ \\
\hline $\begin{array}{l}\text { Number of pulse cycles accounted for } \\
\text { Doppler processing NFFT: } \\
\text { corresponding velocity resolution: }\end{array}$ & $1 \mathrm{~m}$ \\
\hline $\begin{array}{l}\text { Minimal detectable radar reflectivity factor at } \\
\text { range } R, \text { reference range } R_{0}=5 \mathrm{~km},\end{array}$ & $0.56^{\circ}$ (one way, $3 \mathrm{~dB}$ diameter) \\
averaging time $T_{A V}, T_{A V 0}=10 \mathrm{~s}$, and \\
assuming a Doppler peak width of $8 \mathrm{~cm} / \mathrm{s}:$
\end{tabular}


Figure S1. Backscatter profiles from Doppler lidar measured in Kuopio on $10^{\text {th }}$ May 2012. The example shows the change in $\mathrm{ABL}$ data acquisition when telescope focus length was altered from infinite to $2 \mathrm{~km}$ at 10:00.

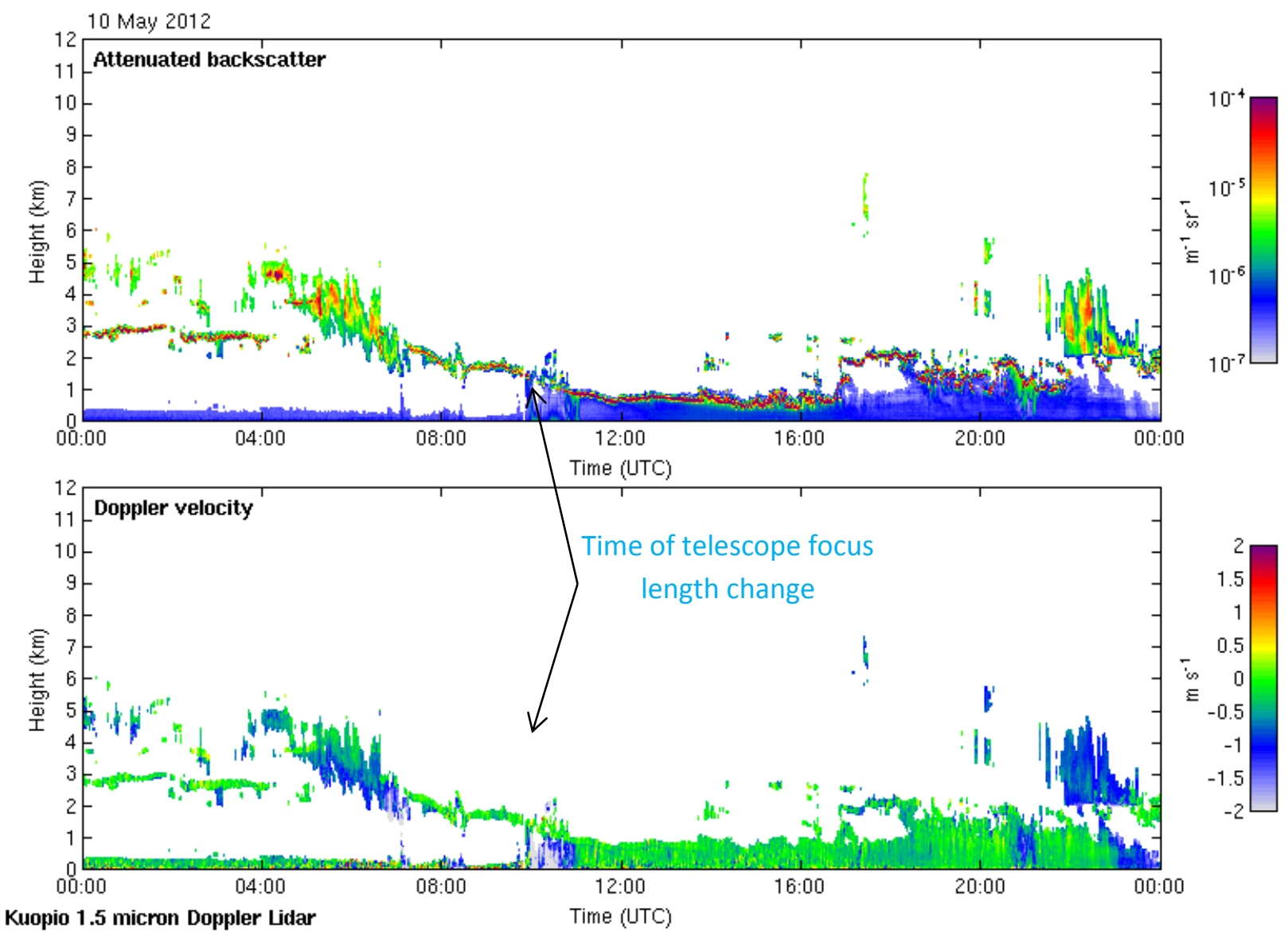


Figure S2. Comparison of wind speed measured with HALO Photonics Doppler lidar and AWSsensor at 220-m height in Kuopio during $20^{\text {th }}$ September 2011-20 ${ }^{\text {th }}$ September 2012. Blue curve is linear fit and green curve is one-to-one line. Statistical analysis of 17494 points resulted in following values: $k=1.08, r=0.84$, rmse $=1.89 \mathrm{~m} \mathrm{~s}^{-1}$.

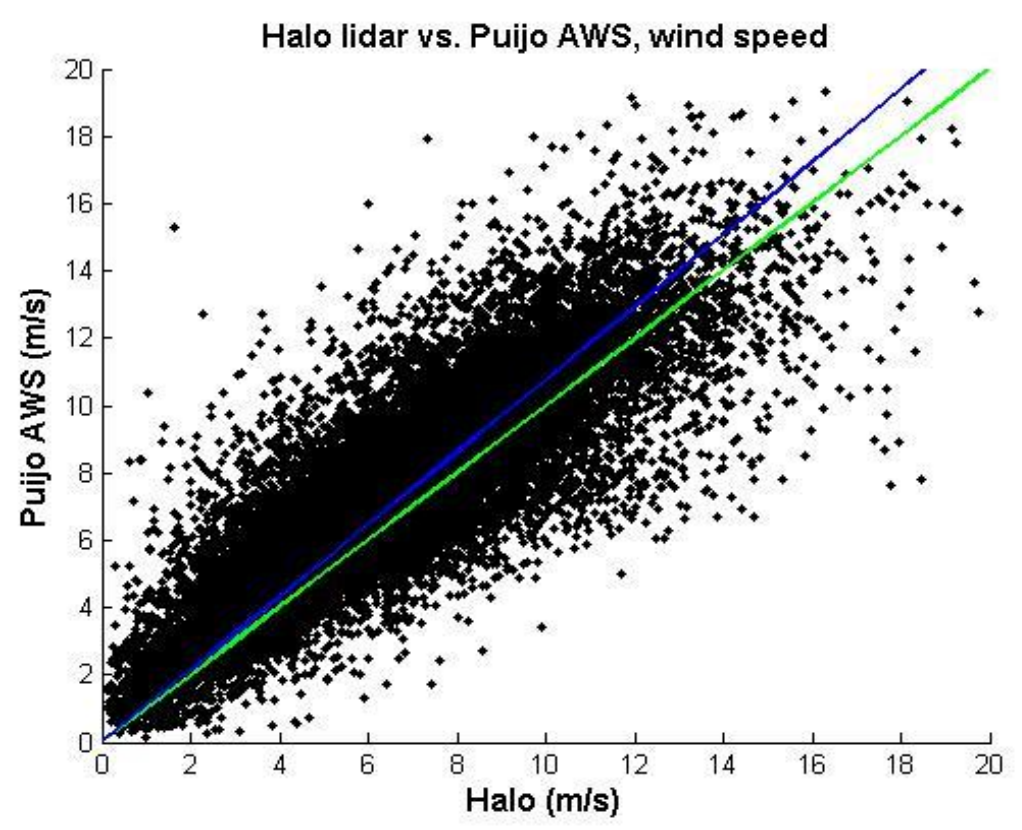

